\title{
Microbial Communities Associated With Stunted Growth Syndrome In Penaeus Vannamei Farming
}

Ashok Kumar Jangam ( $\sim$ ashok.jangam@icar.gov.in )

ICAR-Central Institute of Brackishwater Aquaculture https://orcid.org/0000-0002-2213-2931

\section{Suganya Nathamuni}

ICAR-Central Institute of Brackishwater Aquaculture

Vinaya Kumar Katneni

ICAR-Central Institute of Brackishwater Aquaculture

\section{Satheesha Avunje}

ICAR-Central Institute of Brackishwater Aquaculture

\section{Raymond Jani Angel}

ICAR-Central Institute of Brackishwater Aquaculture

Monendra Grover

ICAR-Indian Agricultural Statistics Research Institute

Mudagandur Shashi Shekhar

ICAR-Central Institute of Brackishwater Aquaculture

\section{Short Report}

Keywords: Metagenomics, stunted growth syndrome, Pacific white shrimp

Posted Date: December 22nd, 2021

DOI: https://doi.org/10.21203/rs.3.rs-1187880/v1

License: (c) (1) This work is licensed under a Creative Commons Attribution 4.0 International License.

Read Full License 


\section{Abstract}

Purpose: Stunted/slow growth syndrome is one of the yield-limiting diseases in Penaeus vannamei farming. Limited information is available on the aetiology of this condition, which needs to be studied to devise prophylactic measures to minimise the production losses. Amongst the factors that influence this condition, microbial communities in the growing environment play an important role. This study aimed at understanding major microbial associations of affected and healthy pond waters through shotgun metagenomics.

Method: The water samples were filtered through vacuum filtration to extract suspended microbes. Subsequently, DNA was isolated from the filtrate using PowerSoil@ DNA Isolation Kit. Libraries prepared from isolated DNA were sequenced using the shotgun metagenomic method on the lllumina HiSeq platform. The microbial profiling and their functional prediction of the shotgun metagenome sequences were carried out using stand-alone versions of Kaiju, OmicsBox respectively.

Results: The taxonomic classification results revealed that species of Oceanospirillum, and vibrio were high in the disease sample, while Rhodobacteraceae bacterium and Neptunomonas were high in the healthy sample. The alpha diversity analysis showed slightly higher diversity in the healthy sample compared to the disease infected. The taxonomic biomarkers for healthy and infected states reported in previous studies were also observed in this study. The major functional associations of both the healthy and infected groups include amino acid transport and metabolism, cell wall/membrane/envelope biogenesis, and energy production and conversion.

Conclusion: The study identified major taxonomical and functional associations of ponds affected and unaffected with stunted growth syndrome. These associations significantly varied between the samples, indicating dysbiosis of the microbial profiles in the pond waters. This dysbiosis could be a potential cause for the manifestation of stunted growth syndrome. Microbial associations along with other pond environmental factors need to be further explored for an in-depth understanding of stunted growth syndrome.

\section{Introduction}

Pond water microbiota plays an important role in nutrient cycling, maintenance of water quality, and health of the farm-reared shrimp (Huang et al., 2018). Significant correlations were reported between rearing-water bacterial communities and the intestinal microbiota of shrimps in a biofloc-based culture system (Cardona et al., 2016). There has been increasing evidence on the role of gut microbiota on important functions like immunity, health regulation, and nutrient absorption in shrimps (Li et al, 2018, Fan et al., 2019, Liu et al., 2019, Servin et al., 2021). Hence, understanding microbial communities present in the culture environment is important in case-control studies to derive causal relations on the manifestation of diseases. 
Pacific white shrimp farming across the world is facing several impediments, mainly due to bacterial and viral diseases. Economic losses due to the diseases in India accounted for an annual loss of US\$1.02 B (Patil et al., 2021). Stunted growth syndrome (SGS) is among one of the most commonly observed diseases contributing to considerable production losses. Genetics, environment, and many infectious agents especially the microsporidian Enterocytozoon hepatopenaei (EHP) were reported to play a role in the manifestation of this disease (Kooloth et al., 2021, Rajendran et al., 2016). However, the exact cause of this multifactor syndrome is unclear, suggesting a more in-depth investigation of all underlying factors.

Microbial communities of bacteria, archaea, viruses play a significant role in pond water quality and shrimp diseases. The study of the ambient microbiota is essential for establishing causal relationships for diseases. However, a major proportion of these microbes is culture-independent and could not be studied through conventional laboratory approaches. In such cases, metagenomics is a game-changer that enables researchers to study the microbial profiles on a larger scale (Quince et al., 2017, Forbes et al., 2017). Metagenomics applications in aquaculture include microbial profiling, diversity analysis, identification of antibiotic resistance genes (ARGs), novel and potential pathogens, microbial associations with bioflocs, and probiotics (Martínez et al., 2017). Studies on microbial associations with shrimp diseases were conducted in recent years on white faeces syndrome (Hou et al., 2018), intestinal disorders (Xiong et al., 2015), Acute hepatopancreatic necrosis disease (AHPND) (Cornejo et al., 2017, Hossain et al., 2021). However, there are no microbial association studies conducted for SGS and this study is the first attempt in this direction.

This study aimed at exploring the possible relationship between microbiota present in rearing water and SGS in shrimp through shotgun metagenomics. Comparative assessment of microbial communities of healthy and disease samples was conducted to understand their presence and functions. Microbial signatures of SGS presented in this study are intended to provide a deeper understanding of the manifestation of this yield-limiting syndrome.

\section{Materials And Methods}

Sample collection

Water samples from Penaeus vannamei culture ponds, both affected and unaffected by SGS, were collected from the farms located at Nellore (Latitude: $14.44 \mathrm{~N}$, Longitude: $79.98 \mathrm{E}$ ) of Andhra Pradesh, India. At the time of collection, ponds were at 70 days of culture and salinity levels were around 23ppt. Both Water and animal samples were collected from the pond experiencing SGS (Disease) and normal ponds (Healthy). Animal samples were tested negative for known pathogens like White spot syndrome virus (WSSV), Infectious hypodermal and hematopoietic necrosis (IHHNV), and Enterocytozoon Hepatopenaei (EHP) (Fig. 1).

Sample preparation, DNA isolation, and sequencing 
Water samples of 10 litres stored at $4^{\circ} \mathrm{C}$ were used for microbial DNA isolation. Five litres of water from infected and healthy samples were centrifuged at $5000 \mathrm{~g}$ for $15 \mathrm{~min}$ at $4^{\circ} \mathrm{C}$. The supernatant was collected in a sterile container. Pellet was suspended in $50 \mathrm{ml}$ sterile seawater and stored at $4^{\circ} \mathrm{C}$ until use. The supernatant was filtered through a $0.22 \mu$ filter paper by vacuum filtration to collect suspended microbes. The filtrate was scrapped and collected in a sterile $2 \mathrm{ml}$ microcentrifuge tube. DNA was isolated from $0.25 \mathrm{~g}$ of microbial samples using the PowerSoil ${ }^{\circledR}$ DNA Isolation Kit (Qiagen). Sequencing libraries were prepared using the NEBnext ultra DNA library preparation kit. The quality of the library was checked using an Agilent TapeStation. Libraries were sequenced using the Illumina HiSeq2500 platform and Paired-end reads of 250 base pairs were generated for both samples.

Taxonomic classification

Raw reads obtained from the sequencer were subjected to different shotgun metagenomic data processing methods like quality assessment and control, taxonomic classification, assembly, differential abundance analysis, functional annotation, diversity analysis, virulence, and resistant gene predictions. Quality control tool FastQC v0.11.8 (Andrews et al., 2010) was used to obtain an overview of the quality metrics of the raw reads. These reads were trimmed for low quality and adapter content using trimmomatic v0.39 (Bolger et al., 2014) and retained sequences having a minimum length of 50 bases. The quality trimmed reads were subjected to taxonomic classification using the standalone version of Kaiju v. 1.7.3 (Menzel et al., 2016). Kaiju was run with default parameters against the latest prokaryotic non-redundant protein database along with fungi and microbial eukaryotes (nr+euk). Microbial diversity analysis was carried out using R library 'vegan' (Oksanen et al., 2013) with a threshold of $0.1 \%$ minimum abundance to estimate Shannon, Simpson, Inverse Simpson and fisher alpha.

Metagenome assembly and annotation

Simultaneously, quality trimmed reads were uploaded to the metagenomics pipeline in OmicsBox (BioBam, 2019) software for functional annotation. OmicsBox implementation of metaSpades v.3.15.2 (Nurk et al., 2017) for metagenome assembly, prodigal (Hyatt et al., 2010) for open reading frames (ORF) prediction and EggNOG (Jensen et al., 2008) mapping for annotation of orthologous groups of genes were chosen in this study. Predicted ORFs were searched against the virulence factor database (VFDB) (Chen et al., 2005) to identify candidate virulent genes with an e-value set at 1e-6. Venn diagram was plotted using the online tool Venn diagram plotting tool (http://bioinformatics.psb.ugent.be/webtools/Venn/).

\section{Data availability}

The nextgen sequencing data of this study are deposited in the SRA repository of Genbank with the accession number PRJNA686191

\section{Results}


Sequence statistics

Shotgun sequencing of the samples generated $8,963,000$ and $8,445,407$ raw sequence reads for both the healthy and disease samples respectively. The quality trimming filtered around $30 \%$ of the reads from both the samples resulting in high quality reads for further processing (Table 1 ). Around $70 \%$ of the reads have been retained post QC, with minimum and maximum read lengths of 50 and 250 for both the healthy and disease groups respectively. GC content of the healthy and diseased samples was found to be $54 \%$ and $45 \%$ respectively.

Taxonomy and diversity indicators

The taxonomic classifications were assigned by Kaiju for $65 \%$ and $74 \%$ reads out of the total reads subjected to analysis for healthy and diseased samples respectively. The classified reads of the healthy sample were composed of $76.4 \%$ of Proteobacteria, $11.6 \%$ of Bacteroidetes, $1.8 \%$ of actinobacteria, whereas the diseased sample was composed of $55.20 \%$ of Proteobacteria, $31.32 \%$ of Bacteroidetes, $3.9 \%$ of Actinobacteria, $2.44 \%$ of Firmicutes, and $2.03 \%$ of Chlamydiae as the dominant or major phyla (Fig. 2a). The families Oceanospirillaceae (20.85\%), Flavobacteriaceae (11.79\%), Crocinitomicaceae (10.90\%), and Vibrionaceae (6.14\%) were highly abundant in the diseased sample, while Rhodobacteraceae (38.5\%) and Pseudoalteromonadaceae (7.6\%) were high in healthy sample. The dominant genera were Pseudoalteromonas (7.6\%), Marivita (4.6\%), Neptunomonas, and Idiomarina (2.0\%) in the healthy and Oceanospirillum (18.72\%), Vibrio (5.87\%), Fluvicola (5.07\%) and Salegentibacter $(4.43 \%)$ in diseased sample (Fig. 2c). Species-level abundances revealed that the healthy sample was dominated by Rhodobacteraceae bacterium EhC02 (15.7\%), Neptunomonas concharum (2.1\%), Idiomarina atlantica (1.5\%), whereas the diseased sample was dominated by Oceanospirillum sactuarii (15.5\%), Vibrio aphrogenes (4.2\%), Fluvicola sp (4.2\%), Salegentibacter mishustinae (1.8\%), and Oceanospirillum maris (1.7\%) (Fig. 2d). A total of 25,028 taxa were found to be common to both the samples, while 6193 taxa are specific to healthy and 2106 are specific to diseased samples (Fig. 3). Complete taxonomic classification lists along with their corresponding read counts are given in supplementary table 1.

The diversity indices to assess the species richness within and between samples were calculated. As shown in table 2, the estimates for Shannon index, Simpson index and fisher alpha were slightly higher for healthy samples compared to diseased samples, which indicates richer diversity in the healthy sample than that of the diseased sample.

Metagenome assembly and annotation

Metagenome assembly carried out with metaSPAdes assembler revealed a total of 16,315 and 8,503 contigs with an N50 value of 5,823 and 12,482 for healthy and diseased groups respectively (Table 3). A total of 91,003 ORFs for healthy and 62,136 for disease sample was predicted from the assembled contigs using the ORF prediction tool Prodigal. 
The EggNOG annotations were assigned to 74,324 predicted ORFs of healthy and 54,077 of disease samples. Out of which 19,575 (healthy) and 17,139 (disease) were assigned with proper GO annotations (Supplementary Table 2). The genes were majorly involved in metabolism in both the samples, especially in amino acid transport \& metabolism, and energy production \& conversion, followed by cellular processing such as defence mechanism, cell wall, membrane and envelope related genes (Fig. 4). Search for antibiotic resistance genes (ARGs) resulted in hits for 147 sequences from healthy samples and 161 sequences from disease samples (Fig. 5). KEGG pathway map IDs were assigned to 29,055 and 22,510 sequences and toxin-antitoxin systems to 328 and 224 sequences for healthy and disease samples respectively. In addition, 197 sequences of healthy and 147 sequences of diseased samples were found to be secretion systems genes of type I, II, III, IV, VI, and VIII.

Virulence factors

A total of 66224 and 53127 hits were generated for healthy and diseased samples respectively from virulence gene prediction through blast against the VFDB database. On considering the hits with a minimum identity percentage of $80 \%$ and query coverage of $40 \%$ a total of 451 hits for healthy and 169 hits for infected samples remained on filtering. On further filtering for the best match with a $100 \%$ query coverage and a minimum of $80 \%$ identity, 18 and 25 putative virulence genes were identified for healthy and diseased samples respectively. The blast results of the predicted ORFs against the VFDB database are provided in Supplementary Table 3.

\section{Discussion}

The optimal quality of the rearing water is essential for the proper growth and development of aquaculture animals, if not managed properly, would act as a source for exerting biotic and abiotic stress and subsequently cause disease (Li et al., 2021). The composition and stability (Dysbiosis) of microbial colonies present in the pond waters are considered to play a major role in disease manifestations (Tello et al., 2020). In the case of shrimp, microbes present in pond waters enter through injury, feed, gills, mouth, and antennal gland (Aguirre-Guzmán et. al., 2010, Liu et al., 2021) and may act as beneficial or detrimental to the host. In recent years, alterations in microbial composition were reported to be the cause of several diseases; however, there are no reports on stunted growth syndrome as of date.

Here, we conducted whole metagenomic profiling for two different shrimp ponds in which one of them was infested with stunted growth syndrome. The absence of known disease-causing pathogens like WSSV, IHHNV and EHP the commonly reported associations to growth retardedness necessitated this study.

Results revealed proteobacteria, bacteroidetes, actinobacteria and firmicutes, the commonly reported dominant phyla in the aquaculture environment (He et al., 2020, Fan, and Li, 2019), are found to be dominant in both samples. Families viz., Rhodobacteraceae and pseudoalteromonadaceae are dominant in the healthy sample, whereas oceanospirillaceae, flavobacteriaceae, and vibrionaceae are dominant in diseased condition. 
A recent study on white faeces syndrome reported a high abundance of Rhodobacteraceae in healthy shrimps compared to slow-growing shrimps and their surroundings (Wang et al., 2020). Other studies reported Rhodobacteraceae and Flavobacteriaceae as the potential taxonomic indicators of shrimp health (Wang et al., 2020; Xiong et al., 2017).

The Oceanospirillum is one of the common inhabitants of marine environments (Leonard et al., 2000). It has been identified to be potentially pathogenic to pacific white shrimp, oysters and humans (Horodesky et al., 2020; Ostrensky et al., 2018; Zhang et al., 2021).

The species-level search revealed a significant difference between healthy and disease samples. The healthy sample showed a high abundance of Rhodobacteraceae bacterium EhC02, while the disease sample was dominated by Oceanospirillum sanctuarii, Oceanospirillum maris, Fluvicola sp., and Vibrio aphrogenes.

Several studies reported lower microbial diversity in disease states in comparison with healthy groups. Here, we observed a similar pattern with regard to alpha diversity indices of SGS samples, even though the difference is marginal. This provides empirical evidence for the beneficial effects of more diverse microbial colonies in the habitat.

The functional profile revealed that genes related to transcriptional regulators, major facilitator superfamily (MFS), proteins conserved in bacteria, acetyltransferase and $A B C$ transporters were found among the top 10 abundant genes in both the samples, in addition to these few antibiotic resistancerelated genes such as resistance nodulation cell division gene was also observed. Here again, the EggNOG annotation also revealed that the functional profile of the diseased sample was associated more with Oceanospirillales and Vibrionales.

\section{Conclusion}

In conclusion, the ambient microbiota showed a considerable difference between healthy and diseased conditions, and changes were observed in both taxonomic and functional profiles. This dysbiosis in the microbiome and its functional profile may be a contributing factor to disease. On the contrary, though the dominant bacteria may not be directly associated with disease, still could be a potential threat when the animal is under stress with their opportunistic disease-causing nature. Future work with better coverage and study of environmental parameters along with biotic composition may provide conclusive results in identifying the causal relationships between microbiota and stunted growth disease. The findings of this study will help in understanding the aetiology of stunted growth syndrome.

\section{List Of Abbreviations}




\begin{tabular}{|ll|}
\hline SGS & Stunted Growth Syndrome \\
\hline WSSV & White Spot Syndrome Virus \\
\hline IHHNV & Infectious Hypodermal and Hematopoietic Necrosis Virus \\
\hline EHP & Enterocytozoon hepatopenaei \\
\hline ORF & Open Reading Frame \\
\hline VFDB & Virulence Factor Database \\
\hline KEGG & Kyoto Encyclopedia of Genes and Genomes \\
\hline
\end{tabular}

\section{Declarations}

\section{Acknowledgements}

The authors are thankful to the funding agency and Director, ICAR-CIBA, for the support provided during this research.

\section{Funding}

This research was funded by the Indian Council of Agricultural Research (ICAR), New Delhi under the Network project on agricultural bioinformatics and computational biology.

\section{Author information}

Affiliations

Genetics and Biotechnology Unit, ICAR-Central Institute of Brackishwater Aquaculture, Chennai, India Ashok Kumar Jangam, Suganya Nathamuni, Vinaya Kumar Katneni, Satheesha Avunje, Raymond Jani Angel, and Mudagandur Shashi Shekhar

Centre for Agricultural Bioinformatics, ICAR-Indian Agricultural Statistics Research Institute. New Delhi, India

Monendra Grover

Authors' contributions

All authors contributed substantially to the paper.

Corresponding author

Correspondence to Ashok Kumar Jangam 


\section{Ethics declarations}

\section{Ethics approval and consent to participate}

This article does not contain any studies with human participants or vertebrate animals performed by any of the authors. The invertebrate animals were handled ethically following the guidelines of the Committee for the Purpose of Control and Supervision of Experiments on Animals (CPCSEA), Govt. of India.

\section{Consent for publication}

All of the authors' consent to the publication of this manuscript in Annals of Microbiology.

\section{Competing interests}

The authors declare that the research was conducted in the absence of any commercial or financial relationships that could be construed as a potential conflict of interest.

\section{References}

1. Andrews S (2010) FastQC: a quality control tool for high throughput sequence data. Version 0.11. 2. https://www.bioinformatics.babraham.ac.uk/projects/fastqc/

2. Bolger AM, Lohse M, Usadel B (2014) Trimmomatic: a flexible trimmer for Illumina sequence data. Bioinformatics 30:2114-2120. https://doi.org/10.1093/bioinformatics/btu170

3. Cardona E, Gueguen Y, Magré K, et al (2016) Bacterial community characterization of water and intestine of the shrimp Litopenaeus stylirostris in a biofloc system. BMC Microbiol 16:157. https://doi.org/10.1186/s12866-016-0770-z

4. Chen L, Yang J, Yu J, et al (2005) VFDB: a reference database for bacterial virulence factors. Nucleic Acids Res 33:D325-D328. https://doi.org/10.1093/NAR/GKI008

5. Cornejo-Granados F, Lopez-Zavala AA, Gallardo-Becerra L, et al (2017) Microbiome of Pacific Whiteleg shrimp reveals differential bacterial community composition between Wild, Aquacultured and AHPND/EMS outbreak conditions. Sci Rep 7:11783. https://doi.org/10.1038/s41598-017-11805w

6. Fan L, Li QX (2019) Characteristics of intestinal microbiota in the Pacific white shrimp Litopenaeus vannamei differing growth performances in the marine cultured environment. Aquaculture 505:450461. https://doi.org/10.1016/j.aquaculture.2019.02.075

7. Fan L, Wang Z, Chen M, et al (2019) Microbiota comparison of Pacific white shrimp intestine and sediment at freshwater and marine cultured environment. Sci Total Environ 657:1194-1204. https://doi.org/10.1016/j.scitotenv.2018.12.069

8. Forbes JD, Knox NC, Ronholm J, et al (2017) Metagenomics: The Next Culture-Independent Game Changer . Front. Microbiol. 8:1069 
9. He Z, Pan L, Zhang M, et al (2020) Metagenomic comparison of structure and function of microbial community between water, effluent and shrimp intestine of higher place Litopenaeus vannamei ponds. J Appl Microbiol 129:243-255. https://doi.org/10.1111/jam.14610

10. HORODESKY A, CASTILHO-WESTPHAL GG, PONT GD, et al (2020) Metagenomic analysis of the bacterial microbiota associated with cultured oysters (Crassostrea sp.) in estuarine environments. An Acad Bras Cienc 92:. https://doi.org/10.1590/0001-3765202020180432

11. Hossain MS, Dai J, Qiu D (2021) Dysbiosis of the shrimp (Penaeus monodon) gut microbiome with AHPND outbreaks revealed by $16 \mathrm{~S}$ rRNA metagenomics analysis. Aquac Res 52:3336-3349. https://doi.org/https://doi.org/10.1111/are.15178

12. Hou D, Huang Z, Zeng $S$, et al (2018) Intestinal bacterial signatures of white feces syndrome in shrimp. Appl Microbiol Biotechnol 102:3701-3709. https://doi.org/10.1007/s00253-018-8855-2

13. Huang F, Pan L, Song M, et al (2018) Microbiota assemblages of water, sediment, and intestine and their associations with environmental factors and shrimp physiological health. Appl Microbiol Biotechnol 102:. https://doi.org/10.1007/s00253-018-9229-5

14. Hyatt D, Chen G-L, LoCascio PF, et al (2010) Prodigal: prokaryotic gene recognition and translation initiation site identification. BMC Bioinformatics 2010111 11:1-11. https://doi.org/10.1186/14712105-11-119

15. J X, J Z, W D, et al (2017) Integrating gut microbiota immaturity and disease-discriminatory taxa to diagnose the initiation and severity of shrimp disease. Environ Microbiol 19:1490-1501. https://doi.org/10.1111/1462-2920.13701

16. Jensen LJ, Julien P, Kuhn M, et al (2008) eggNOG: automated construction and annotation of orthologous groups of genes. Nucleic Acids Res 36:D250-D254. https://doi.org/10.1093/NAR/GKM796

17. Leonard N, Blancheton JP, Guiraud JP (2000) Populations of heterotrophic bacteria in an experimental recirculating aquaculture system. Aquac Eng 22:109-120. https://doi.org/10.1016/S0144-8609(00)00035-2

18. Li E, Xu C, Wang X, et al (2018) Gut Microbiota and its Modulation for Healthy Farming of PacifiC White Shrimp Litopenaeus vannamei. Rev Fish Sci Aquac 26:381-399. https://doi.org/10.1080/23308249.2018.1440530

19. Li J, Fang L, Liang XF, et al (2021) Influence of environmental factors and bacterial community diversity in pond water on health of Chinese perch through Gut Microbiota change. Aquac Reports 20:100629. https://doi.org/10.1016/J.AQREP.2021.100629

20. Liu Q, Lai Z, Gao Y, et al (2021) Connection between the Gut Microbiota of Largemouth Bass (Micropterus salmoides) and Microbiota of the Pond Culture Environment. Microorg 2021, Vol 9 , Page 1770 9:1770. https://doi.org/10.3390/MICROORGANISMS9081770

21. Martínez-Porchas $M$, Vargas-Albores $F$ (2017) Microbial metagenomics in aquaculture: a potential tool for a deeper insight into the activity. Rev Aquac 9:42-56. https://doi.org/https://doi.org/10.1111/raq.12102 
22. Menzel P, Ng KL, Krogh A (2016) Fast and sensitive taxonomic classification for metagenomics with Kaiju. Nat Commun 7:1-9. https://doi.org/10.1038/ncomms11257

23. Nurk S, Meleshko D, Korobeynikov A, Pevzner PA (2017) metaSPAdes: a new versatile metagenomic assembler. Genome Res 27:824-834. https://doi.org/10.1101/GR.213959.116

24. Oksanen J, Blanchet FG, Kindt R, et al (2013) Package 'vegan.' Community Ecol Packag version 2:1295

25. Ostrensky A, Horodesky A, Faoro $\mathrm{H}$, et al (2018) Metagenomic evaluation of the effects of storage conditions on the bacterial microbiota of oysters Crassostrea gasar (Adanson, 1757). J Appl Microbiol 125:1435-1443. https://doi.org/10.1111/JAM.14045

26. Patil PK, Geetha R, Ravisankar T, et al (2021) Economic loss due to diseases in Indian shrimp farming with special reference to Enterocytozoon hepatopenaei (EHP) and white spot syndrome virus (WSSV). Aquaculture 533:736231.

https://doi.org/https://doi.org/10.1016/j.aquaculture.2020.736231

27. Quince C, Walker AW, Simpson JT, et al (2017) Shotgun metagenomics, from sampling to analysis. Nat Biotechnol 35:833-844. https://doi.org/10.1038/nbt.3935

28. Rajendran K V., Shivam S, Ezhil Praveena P, et al (2016) Emergence of Enterocytozoon hepatopenaei (EHP) in farmed Penaeus (Litopenaeus) vannamei in India. Aquaculture 454:272-280. https://doi.org/10.1016/J.AQUACULTURE.2015.12.034

29. Servin Arce K, de Souza Valente C, do Vale Pereira G, et al (2021) Modulation of the gut microbiota of Pacific white shrimp (Penaeus vannamei Boone, 1931) by dietary inclusion of a functional yeast cell wall-based additive. Aquac Nutr 27:1114-1127. https://doi.org/https://doi.org/10.1111/anu.13252

30. Tello M, Valdes N, Vargas R, et al (2020) Application of Metagenomics to Chilean Aquaculture. In: Metagenomics - Basics, Methods and Applications. IntechOpen

31. Valappil RK, Stentiford GD, Bass D (2021) The rise of the syndrome - sub-optimal growth disorders in farmed shrimp. Rev Aquac 13:1888-1906. https://doi.org/10.1111/RAQ.12550

32. Wang H, Wan X, Xie G, et al (2020a) Insights into the histopathology and microbiome of Pacific white shrimp, Penaeus vannamei, suffering from white feces syndrome. Aquaculture 527:735447. https://doi.org/10.1016/J.AQUACULTURE.2020.735447

33. Wang S, Yan Z, Wang P, et al (2020b) Comparative metagenomics reveals the microbial diversity and metabolic potentials in the sediments and surrounding seawaters of Qinhuangdao mariculture area. PLoS One 15:1-16. https://doi.org/10.1371/journal.pone.0234128

34. Xiong J, Wang K, Wu J, et al (2015) Changes in intestinal bacterial communities are closely associated with shrimp disease severity. Appl Microbiol Biotechnol 99:6911-6919. https://doi.org/10.1007/s00253-015-6632-z

35. Xiong J, Zhu J, Dai W, et al (2017) Integrating gut microbiota immaturity and disease-discriminatory taxa to diagnose the initiation and severity of shrimp disease. Environ Microbiol 19:1490-1501. https://doi.org/10.1111/1462-2920.13701 
36. Zhang X, Sun J, Han Z, et al (2021) Vibrio parahaemolyticus alters the community composition and function of intestinal microbiota in Pacific white shrimp, Penaeus vannamei. Aquaculture 544:737061. https://doi.org/10.1016/J.AQUACULTURE.2021.737061

37. Zhao Y, Duan C, Zhang X, et al (2018a) Insights into the Gut Microbiota of Freshwater Shrimp and Its Associations with the Surrounding Microbiota and Environmental Factors. J Microbiol Biotechnol 28:946-956. https://doi.org/10.4014/JMB.1709.09070

38. Zhao $Y$, Zhang $X$ xiang, Zhao Z, et al (2018b) Metagenomic analysis revealed the prevalence of antibiotic resistance genes in the gut and living environment of freshwater shrimp. J Hazard Mater 350:10-18. https://doi.org/10.1016/J.JHAZMAT.2018.02.004

39. Zoqratt MZHM, Eng WWH, Thai BT, et al (2018) Microbiome analysis of Pacific white shrimp gut and rearing water from Malaysia and Vietnam: implications for aquaculture research and management. PeerJ 6:e5826. https://doi.org/10.7717/PEERJ.5826

\section{Tables}

Table 1: Sequence statistics

\begin{tabular}{lll} 
& Healthy & Disease \\
\hline Raw reads & 8963000 & 8445407 \\
\hline Clean Reads & 6291889 & 5856013 \\
\hline Sequence Length bp & 250 & 250 \\
\hline Sequence Length of clean reads bp & $50-250$ & $50-250$ \\
\hline GC (\%) & 54 & 45
\end{tabular}

Table 2: Alpha diversity indices values for the species level taxonomic abundance

\begin{tabular}{lll} 
Index & Healthy & Disease \\
\hline Shannon & 6.37 & 5.48 \\
\hline Simpson & 0.95 & 0.94 \\
\hline Inverse Simpson & 18.56 & 16.90 \\
\hline Fisher Alpha & 4411.01 & 3454.55
\end{tabular}

Table 3: Metagenome assembly and annotation statistics 


\begin{tabular}{lll} 
Assembler & Diseased & Healthy \\
\hline Number of Contigs & 8503 & 16315 \\
\hline Longest Contig (bp) & 1304375 & 719730 \\
\hline N50 & 12482 & 5823 \\
\hline L50 & 695 & 7627 \\
\hline Min length & 2000 & 2000 \\
\hline Max length & 1304375 & 719730 \\
\hline Average length & 6916.87 & 5092.68 \\
\hline Identified ORFs & 62136 & 91003 \\
\hline Number of GO annotated sequences & $17139 / 27.58 \%$ & $19575 / 21.51 \%$ \\
\hline Number of GO annotations & 88195 & 120172
\end{tabular}

\section{Figures}




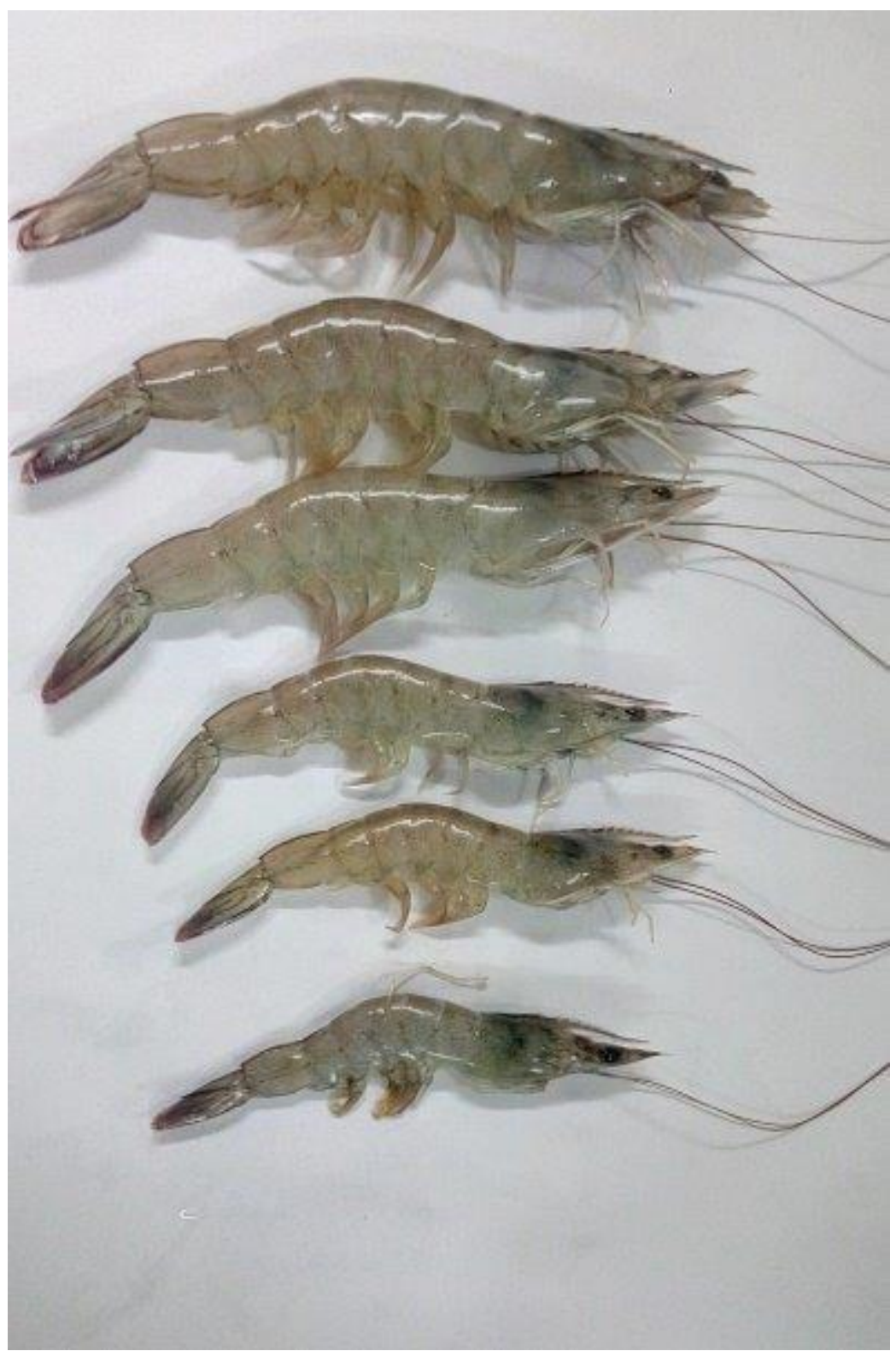

\section{Figure 1}

The above image shows the variation in size of shrimps from the same pond that had been affected by stunted growth. 
(a) Phylum level

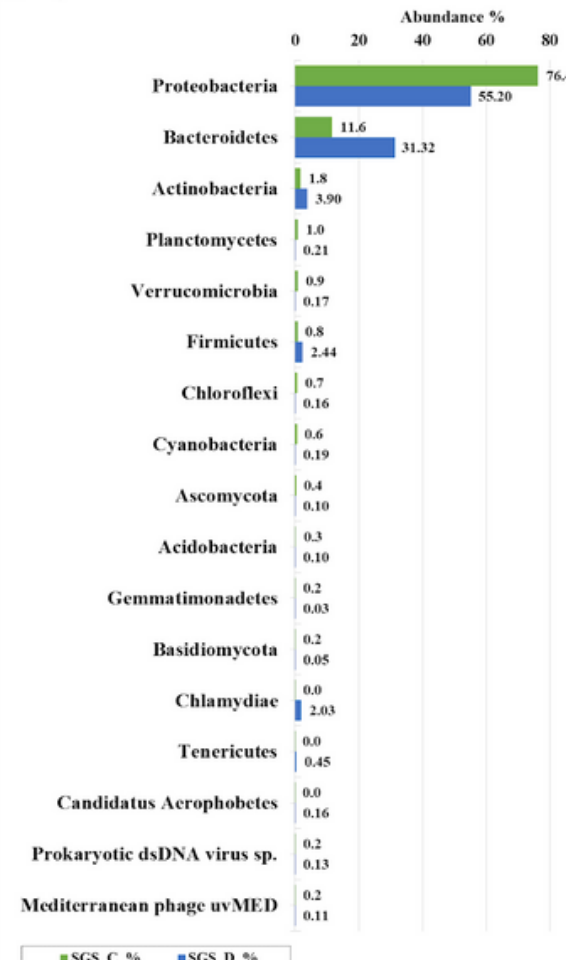

(b) Genus level

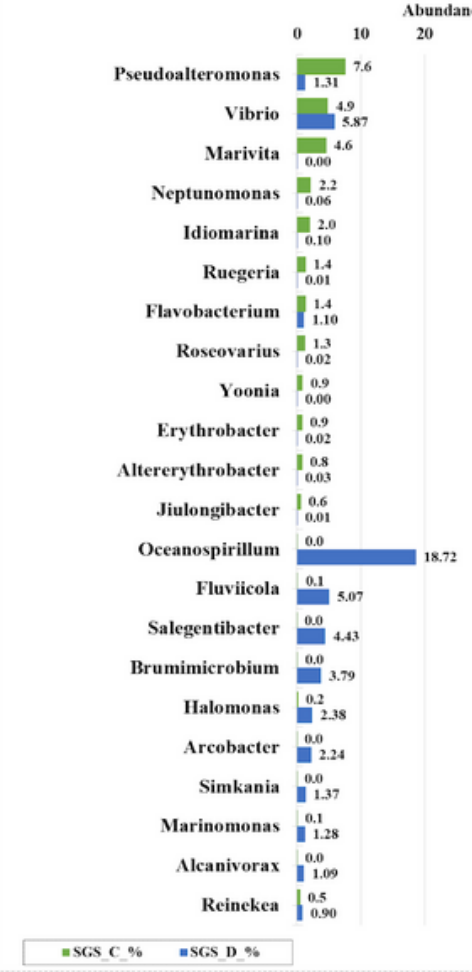

(b) Family level

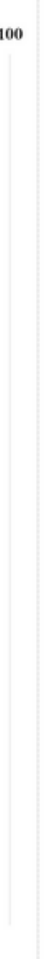

(b) Species level
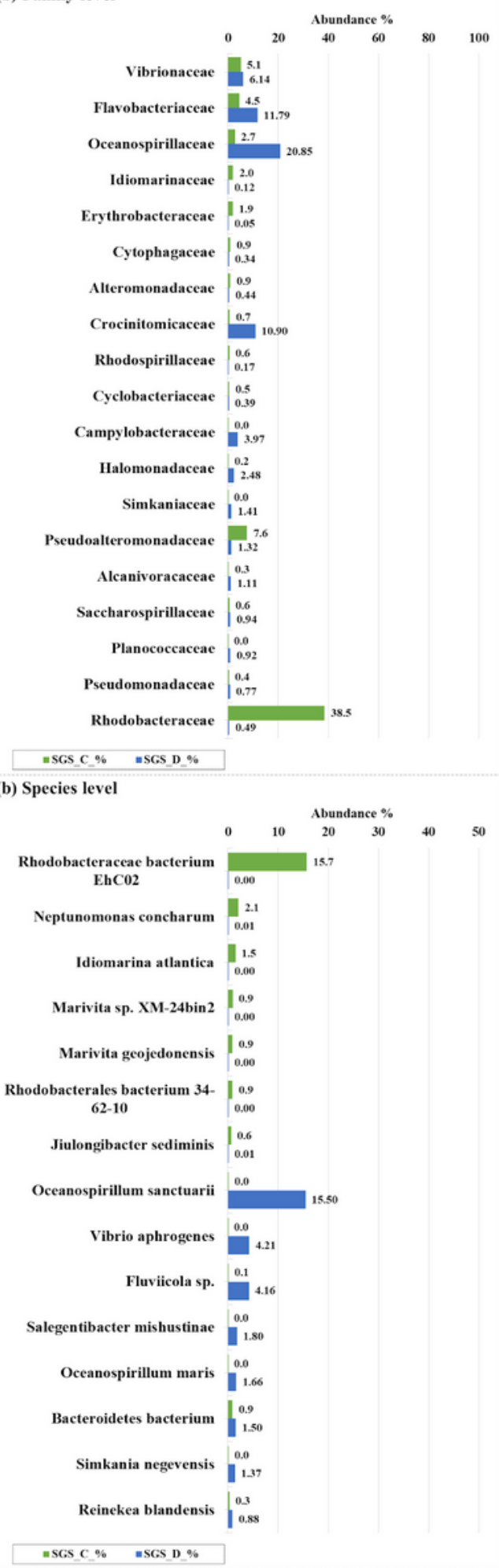

\section{Figure 2}

The bar graphs represent the highly abundant taxa for each taxonomic level. (a)Phylum level (b) Family level (c) Genus level and (d) Species-level abundance. 


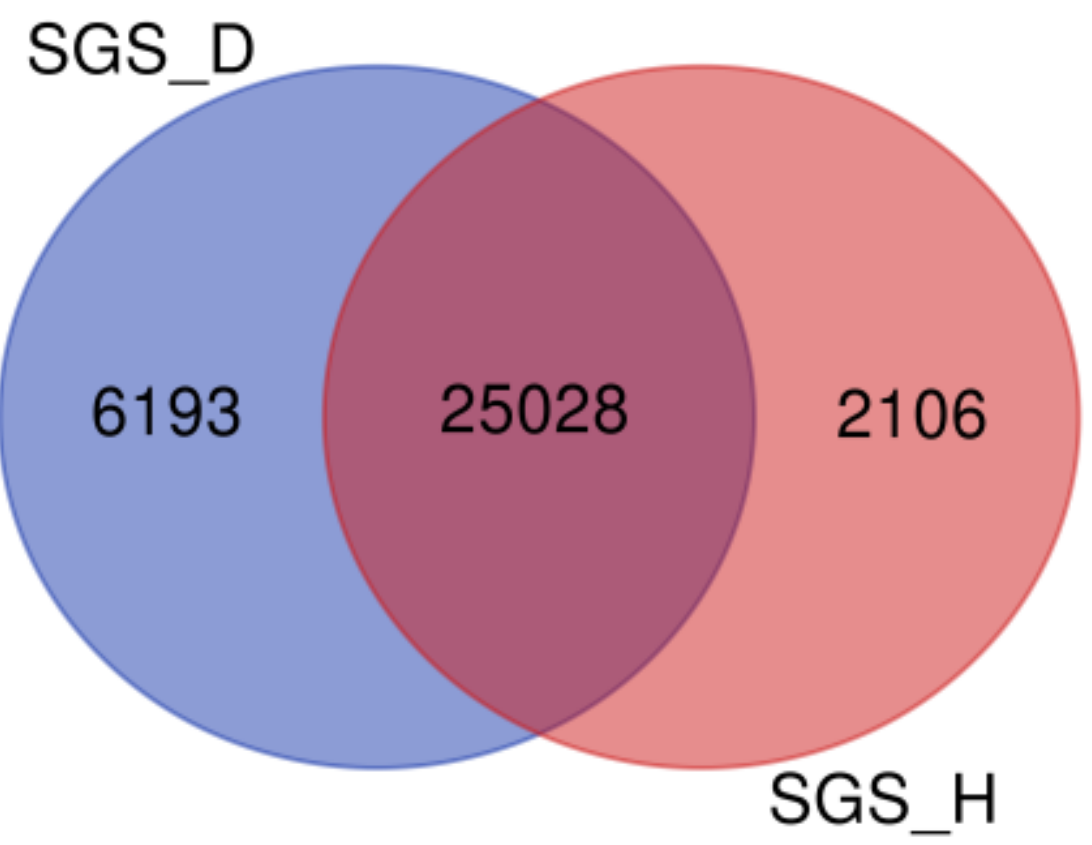

Figure 3

Venn diagram showing the quantitative taxa composition of the two samples. 


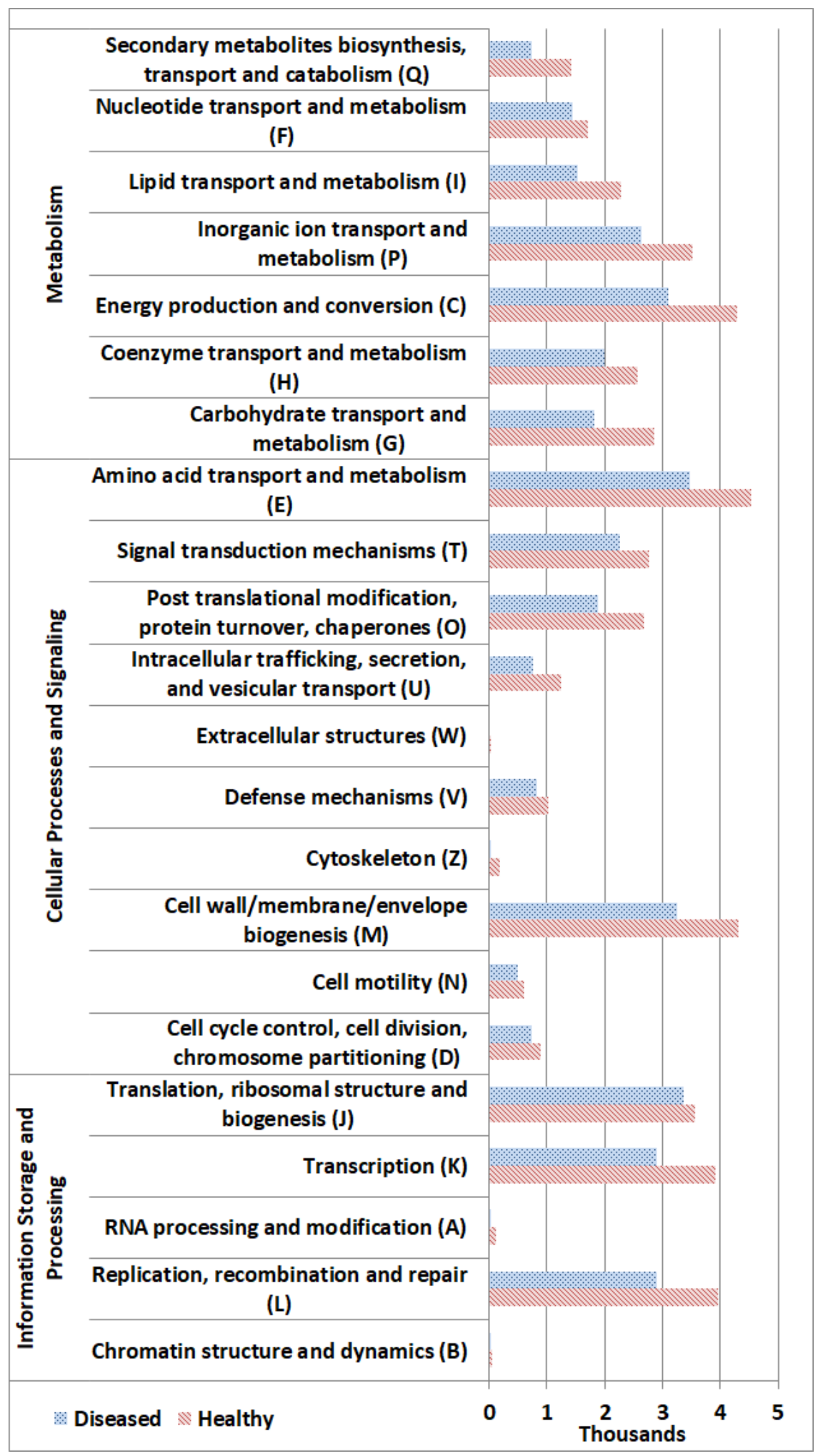

\section{Figure 4}

Distribution of COG categories among healthy and disease samples 

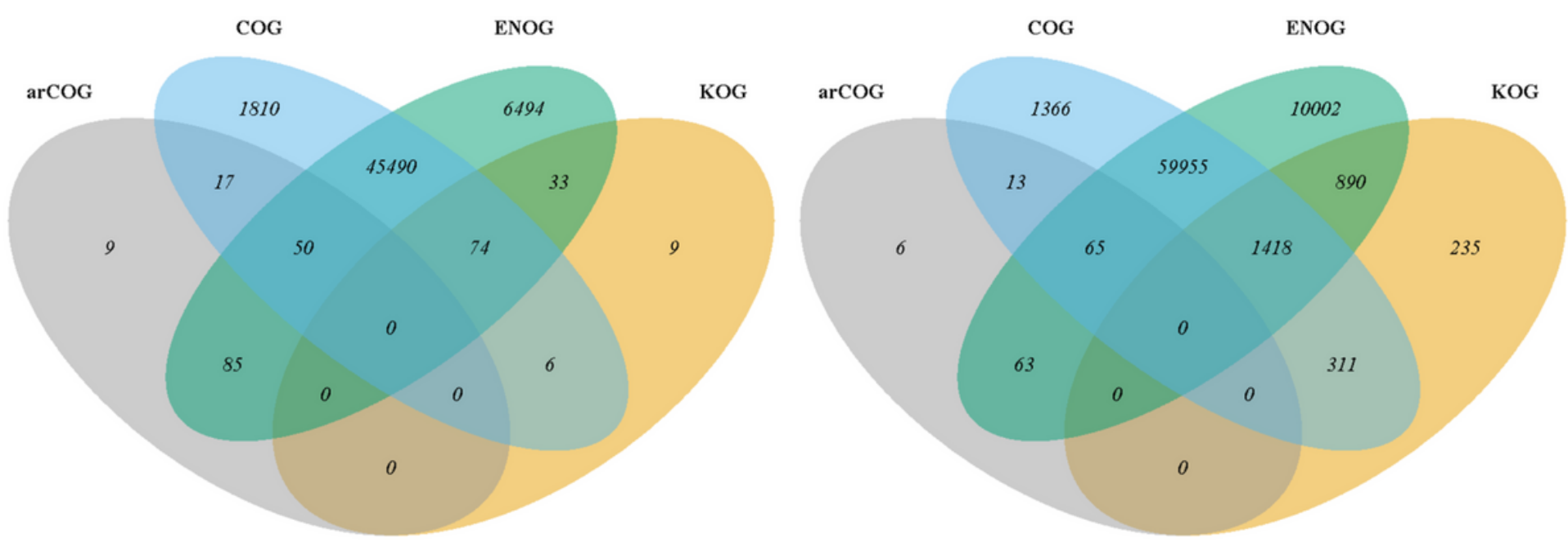

\section{Figure 5}

Venn diagram showing quantitative results of the EggNOG annotation pipeline.

\section{Supplementary Files}

This is a list of supplementary files associated with this preprint. Click to download.

- SupplementaryTable1.xlsx

- SupplementaryTable2.xlsx

- SupplementaryTable3.xlsx 